\title{
Primary angiitis of central nervous system: The story of a great masquerader
}

\author{
Bhupender Kumar Bajaj, Shweta Pandey, Bhargavi Ramanujam, Ankur Wadhwa \\ Department of Neurology, Post Graduate Institute of Medical Education and Research (PGIMER), Dr. Ram Manohar Lohia Hospital, \\ New Delhi, India
}

\begin{abstract}
Primary angiitis of central nervous system (PACNS) is characterized by non-caseating granulomatous angiitis restricted to CNS. The condition often masquerades as migraine, stroke, epilepsy, dementia, demyelinating disorder and CNS infection. The protean manifestations frequently lead to misdiagnoses. We present a case of a young male from rural background that remained undiagnosed for years as the possibility of PACNS was not considered. He had history suggestive of migraine-like headaches followed by seizures. Subsequently, he developed rapidly progressive dementia and two episodes of hemorrhagic strokes over a short period. The diagnosis was finally clinched by the absence of evidence of systemic vasculitis and the presence of characteristic non-caseating granuloma around vessels of duramater and cerebral parenchyma on brain biopsy. He was started on pulse therapy with intravenous cyclophosphamide and methylprednisolone. The current literature about the condition and its management is reviewed in this report.
\end{abstract}

Key words: Central nervous system, primary angiitis, vasculitis

\section{Introduction}

Central nervous system (CNS) may be affected by vasculitides as part of systemic illness or the vasculitic process may afflict the CNS in isolation. The latter is called primary angiitis of central nervous system (PACNS). The condition has protean manifestations. Patients with the angiitis may present with ischemic and/or hemorrhage stroke, progressive cognitive impairment, parkinsonism, ataxia, ocular movement abnormalities, dysarthria, dysphonia, dysphagia, myelopathy, headaches, seizures and other manifestations..$^{[1,2]}$ There is as yet little information available about etiopathogenesis and management of the condition. Delay in diagnosis can lead to irreversible neurologic deficits. We present a case of a male patient in whom the disease masqueraded as various other conditions until finally PACNS was diagnosed.

\begin{tabular}{|l|l|}
\hline \multicolumn{2}{|c|}{ Access this article online } \\
\hline Quick Response Code: & Website: \\
\hline & www.ruralneuropractice.com \\
\hline & \\
\hline
\end{tabular}

\section{Case Report}

A 28-year-old non-hypertensive, non-diabetic, male tailor presented with recurrent episodic hemicranial throbbing headache associated with nausea for 4 years and generalized tonic-clonic seizures for around 1 year. His CT scan head showed multiple white matter hypodensities with evidence of speckled enhancement in some areas post contrast [Figure 1]. Provisional diagnosis of epilepsy due to multiple neurocysticercosis and migraine was considered and the patient was started on antiepileptic drug, phenytoin. While his brain MRI was being planned, he became clumsy, inattentive and started taking extraordinarily long time to tailor-cut the cloth material. He lost his job because of his inability to properly cut and sew clothes. He became duller in performing routine chores at home. Meanwhile, MRI brain showed multiple white matter T1W hypointensities and $\mathrm{T} 2 \mathrm{~W}$ hyperintensities with evidence of speckled enhancement post-gadolinium [Figure 1]. He started having difficulty in seeing and recognizing objects and people. His fundus examination did not reveal any abnormality and his pupillary light responses were noted to be normal. Visual evoked potential studies of the patient showed prolonged P100 latencies. MR angiography (MRA) of intracranial and neck vessels did

Address for correspondence:

Dr. Bhupender Kumar Bajaj, Department of Neurology, Post Graduate Institute of Medical Education and Research (PGIMER), Dr. Ram Manohar Lohia Hospital, Baba Kharag Singh Marg, New Delhi - 110 001, India. E-mail: docbajaj@yahoo.co.in 
not reveal any vascular anomaly. The patient was lost to follow-up over the next few months. During this time, he wandered away from his home without being able to find his way back. When he was traced subsequently to a city away from his home, he could not recall what transpired during the days. He was noted to have become forgetful about recent events. Subsequently, he had convulsions and developed acute onset right hemiparesis resulting in his fall from stairs. He was admitted to another local medical facility and his CT scan head done there showed left frontal hematoma. He improved only to have recurrence of sudden onset weakness of right half of body with aphasia following an episode of seizure and was admitted in our hospital. Brain CT scan showed resolving hematoma in the left frontal region. In the hospital, the patient developed acute confusional state and a repeat CT scan showed appearance of fresh right sided frontal parenchymal hematoma. After re-evaluation of patient's history and imaging findings, the diagnosis of CNS angiitis was considered. The patient tested negative for various investigations including antinuclear antibody, rheumatoid arthritis factor, cytoplasmic and perinuclear antineutrophilic cytoplasmic antibody, venereal disease research laboratory test for syphilis and ELISA for human immunodeficiency virus. Cerebrospinal fluid (CSF) analysis of the patient was done once the patient stabilized and it showed raised protein levels (111 $\mathrm{mg} \%$; normal range: $15-45 \mathrm{mg} \%)$, normal glucose (52 $\mathrm{mg} \%$ normal range $45-80 \mathrm{mg} \%$ ) with normal cytology. Gram stain, ZN stain, India ink stain and culture of CSF were negative. Oligoclonal bands in CSF were found to be positive. The biopsy of brain showed non-caseating granuloma in the dura and brain parenchyma confirming the diagnosis of granulomatous angiitis of CNS [Figure 2]. The patient was given pulse therapy of intravenous methylprednisolone and cyclophosphamide. Pulse of cyclophosphamide was repeated twice before the patient was lost in the follow up. On his last hospital

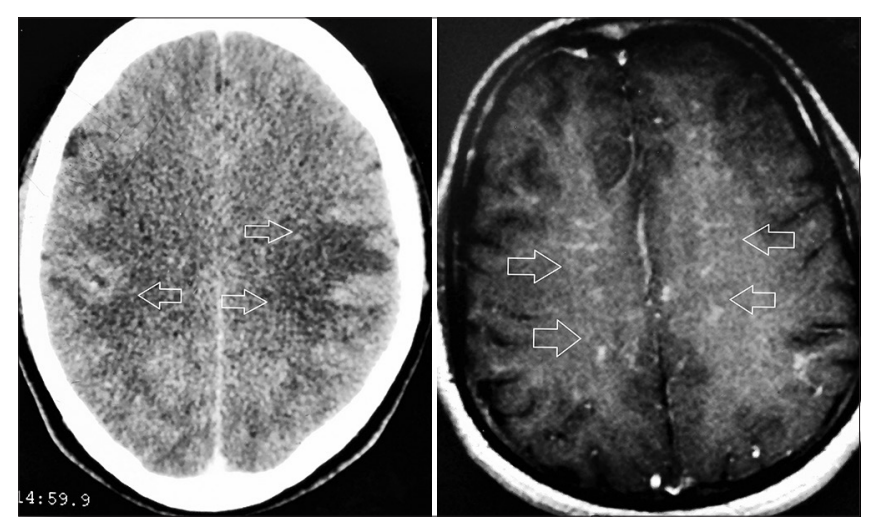

Figure 1: Bilateral frontal and parietal hypodensities (arrows) seen on brain CT scan (Contrast enhanced) on the left. These appear as iso or hypointese lesions exhibiting speckled enhancement on MRI brain (with gadolinium enhancement) appearance, he had not shown any signs and symptoms of deterioration or improvement.

\section{Discussion}

PACNS is a diagnostic challenge. The median age of onset of the condition is about 50 years. Neurological manifestations are diverse, the most common being headache, cognitive impairment, ataxia, seizures, and focal neurological deficits such as hemiparesis, aphasia, dysarthria, decreased visual acuity due to transient ischemic attack, ischemic stroke and intracranial hemorrhage. The cause and pathogenesis of primary CNS vasculitis are unknown. Focal neurological manifestations, headache and seizures are present in various other disorders affecting cerebral blood vessels. Reversible cerebral vasoconstriction syndrome (RCVS) is a common mimicker of PACNS. The condition characterized by vasoconstriction of cerebral vessels is seen in postpartum state and due to exposure to vasoactive drugs. ${ }^{[3]}$ Cerebral angiography shows segmental vasoconstriction of cerebral blood vessels similar to the appearance in PACNS. The condition is not associated with inflammatory changes of the vessels as are seen in PACNS. Repeat cerebral angiography shows resolution of the changes within 12 weeks of the presentation. Another important differential diagnosis is systemic vasculitis which is commonly associated with the presence of constitutional symptoms and serological markers. Our patient initially had history of recurrent migraine-like headaches and generalized convulsions. He subsequently had 2 episodes of acute hemorrhagic stroke over a period of 2 months. The patient developed insidious onset cognitive dysfunction involving multiple domains including attention, recent

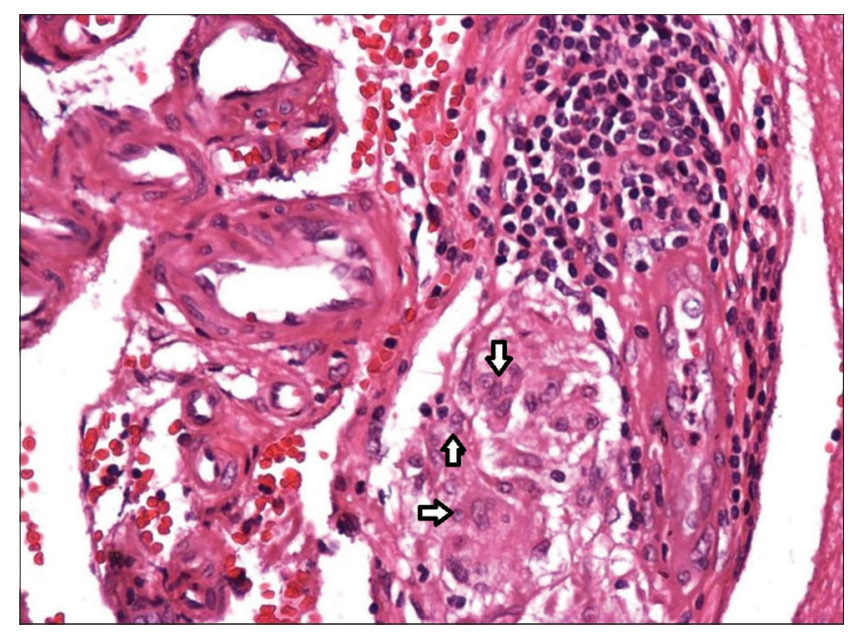

Figure 2: Histopathological appearance of brain/meningeal biopsy showing giant cells and mononuclear cell infiltration of vessel walls in the meninges ( $\mathrm{H}$ and $\mathrm{E}$ stain) 
memory, judgment, visuospatial orientation, language function and praxis. Intracranial hemorrhage followed by subarachnoid hemorrhage are the presenting feature in $11-12 \%$ of PACNS. ${ }^{[4]}$ Subarachnoid hemorrhage is more common than parenchymal hemorrhage in RCVS. Compared to patients without intracranial hemorrhage, those with it are reportedly less likely to have altered cognitive function and require therapy less frequently on subsequent follow-up. The hallmark of the PACNS is that the inflammatory process is limited to the CNS. Sensitivity of cerebral angiography for detection of vasculitis in various studies is between $27 \%$ and $90 \%$ and for brain biopsy it is between $36 \%$ and $83 \% .{ }^{[5]}$ Brain biopsy and histopathological examination is the gold standard diagnostic test. Granulomatous, lymphocytic and necrotizing vasculitis are the three main histopathological patterns of PACNS. ${ }^{[4,6]}$ The classical findings suggestive of granulomatous angiitis are observed in around $50-60 \%$ of cases. Lymphocytic and necrotizing patterns are observed in $25 \%$ and $14-22 \%$ respectively of cases of PACNS. Necrotizing vasculitis is characterized by fibrinoid necrosis and commonly presents with hemorrhage. The inflammation and fibrinoid necrosis leads to thickening of vessel walls which increases the risk of vascular rupture and aneurysmal dilatation. The different histopathological patterns may coexist in some patients. Although there are no controlled therapeutic trials for CNS vasculitis, current treatment recommendation is steroid along with intravenous cyclophosphamide for severe disease. ${ }^{[7]}$ The combination of corticosteroid and rituximab was recently reported to be effective in a report of patient who did not tolerate cyclophosphamide. ${ }^{[8]}$

As yet, there is scarcity of information about etiology, pathogenesis, appropriate diagnostic strategies and treatment approaches for PACNS.

\section{References}

1. Salvarani C, Brown RD Jr, Calamia KT, Christianson TJ, Weigand SD, Miller DV, et al. Primary central nervous system vasculitis: Analysis of 101 patients. Ann Neurol 2007:62:442-51.

2. Hajj-Ali RA, Calabrese LH. Diagnosis and classification of central nervous system vasculitis. J Autoimmun 2014;48-49:149-52.

3. Calabrese LH, Dodick DW, Schwedt TJ, Singhal AB. Narrative review: Reversible cerebral vasoconstriction syndromes. Ann Intern Med 2007;146:34-44.

4. Calabrese LH, Duna GF, Lie JT. Vasculitis in the central nervous system. Arthritis Rheum 1997;40:1189-201.

5. Hajj-Ali RA, Singhal AB, Benseler S, Molloy E, Calabrese LH. Primary angiitis of the CNS. The Lancet Neurol 2011;10:561-72.

6. Salvarani C, Brown RD Jr, Calamia KT, Christianson TJ, Huston J $3^{\text {rd }}$, Meschia JF, et al. Primary central nervous system vasculitis presenting with intracranial hemorrhage. Arthritis Rheum 2011;63:3598-606.

7. Berlit P. Diagnosis and treatment of cerebral vasculitis. Ther Adv Neurol Disord 2010;3:29-42.

8. Salvarani C, Brown RD Jr, Huston J $3^{\text {rd }}$, Morris JM, Hunder GG. Treatment of primary CNS vasculitis with rituximab: Case report. Neurology 2014;82:1287-8.

How to cite this article: Bajaj BK, Pandey S, Ramanujam B, Wadhwa A. Primary angiitis of central nervous system: The story of a great masquerader. J Neurosci Rural Pract 2015;6:399-400.

Source of Support: Nil. Conflict of Interest: None declared. 